Meta

Journal des traducteurs

Translators' Journal

\title{
La rédaction des rapports
}

\section{David Fortin}

Volume 11, numéro 1, mars 1966

URI : https://id.erudit.org/iderudit/002763ar

DOI : https://doi.org/10.7202/002763ar

Aller au sommaire du numéro

Éditeur(s)

Les Presses de l'Université de Montréal

ISSN

0026-0452 (imprimé)

1492-1421 (numérique)

Découvrir la revue

Citer ce document

Fortin, D. (1966). La rédaction des rapports. Meta, 11(1), 32-40.

https://doi.org/10.7202/002763ar

Ce document est protégé par la loi sur le droit d'auteur. L’utilisation des services d'Érudit (y compris la reproduction) est assujettie à sa politique d'utilisation que vous pouvez consulter en ligne.

https://apropos.erudit.org/fr/usagers/politique-dutilisation/
Cet article est diffusé et préservé par Érudit.

Érudit est un consortium interuniversitaire sans but lucratif composé de l’Université de Montréal, l'Université Laval et l'Université du Québec à Montréal. Il a pour mission la promotion et la valorisation de la recherche. https://www.erudit.org/fr/ 


\section{LES \\ OUTILS \\ DU \\ TRADUCTEUR}

\section{LA RÉDACTION DES RAPPORTS}

\section{Éléments de bibliographie réunis à l'intention des rédacteurs des services gouvernementaux}

Le traducteur de l'administration fédérale canadienne est souvent auss. préparateur de manuserits pour l'impression, dès que les textes qu'il traduit sont destinés à une grande diffusion. C'est ce qui nous a porté à surveiller constamment la parution de tous les ouvrages et publications qui touchent de près ou de loin la rédaction et l'élaboration des imprimés ayant un caractère officiel.

Nous avons done réuni graduellement plusieurs titres d'ouvrages qui semblaient utiles aux traducteurs d'abord mais aussi à tous ceux qui doivent, pendant la totalité ou une partie de leur temps, faire de la rédaction administrative, technique ou scientifique, pour un service de l'État.

La liste qui suit ne constitue pas une bibliographie critique. Elle est forcément incomplète et un peu arbitraire dans le choix des ouvrages. Nous classons rubriques et ouvrages par ordre alphabétique en poussant la division aussi loin que possible afin de faciliter la consultation. Le plan de la bibliographie est le suivant, les mots en majuscules indiquant l'ordre alphabétique employé:

CLASSEMENT - Catalogues

Organisation des CONGRES

CORRESPONDANCE

DICTIONNAIRES et Grammaires

LINGUISTIQUE

Élaboration des PÉRIODIQUES

et autres publications

- Abréviation des titres

- Manchette bibliographique

- Ouvrages généraux

- Présentation des articles
- Présentation des périodiques

- Références bibliographiques

- Sommaires, Analyses et Résumés

PROTOCOLE

Rédaction des RAPPORTS

STYLE ADMINISTRATIF

STYLE JUDICIAIRE

TRANSLITTERATION

TYPOGRAPHIE - Présentation des manuserits

UNITÉS et Symboles

VULGARISATION AGRICOLE 
Ce qui nous désappointe en examinant des publications canadiennes du même ordre, c'est de ne pas $y$ trouver mention de textes fondamentaux tels que le Code international d'abréviations de titres de périodiques, publié en 1930 par l'Institut international de coopération intellectuelle; les normes et recommandations qui ont remplacé le Code et qui traitent de l'abréviation des titres et de la présentation des périodiques en général; le Code typographique, enfin, dont la première édition remonte pourtant à 1926. L'isolement dans lequel le Canadien français travaille explique sans doute pour une bonne part le fait que ces publications semblent si peu connues au Canada. Quoi qu'il en soit, il y a lieu, à notre avis, de faire un effort spécial pour trouver les travaux de ce genre et les signaler sans tarder à l'attention du public.

\section{CLASSEMENT — Catalogues}

Assoctation française de noRmalisation (AFNOR), Norme NF Z 44-001 Règles du répertoire alphabétique français. Paris, juin 1943. [21 × $27^{1}$, 5 p.] Complétée par 1'Additif PN Z 44-001, Classement des rubriques avant leurs mots directeurs homographes. Mai 1950. [1 p.]

- , Norme NF Z 44-050 - Les Catalogues alphabétiques d'auteurs et d'anonymes - Rédaction de la notice catalographique. Janvier 1957. [32 p.]

- , Norme NF Z 44-060 - Les Catalogues alphabétiques d'auteurs et d'anonymes - Choix des vedettes - Collectivités-auteurs. Comprend une annexe relative aux congrès. Octobre 1955. [18 p.]

- , Norme NF Z 44-070 - Catalogue alphabétique de matières. Avril 1957. [14 p.]

\section{Organisation des CONGRES}

Duchesne, Lucien-R., Manuel de l'organisateur de congrès. «La Science des congrès 》, Bruxelles, Union des associations internationales, 1960. [20 x $25,135 \mathrm{p}$.]

Renferme entre autres des modèles de divers imprimés essentiels dans l'organisation des congrès.

MarSDen, A. W., The Organization of International Congresses. Dans Laboratory Practice, 11.11 (1962) : 821-826.

Readett, Alan G., Preparations for an International Conference. Monograph No. 1, Londres, The Institute of Linguists, 1963. [14 x 21.5, 20 p.]

UNION DES ASSOCIATIONS INTERNATIONALES, Théorie et Pratique de l'organisation des congrès internationaux. «La Science des congrès», Bruxelles, Union des associations internationales, 1960. [20 x 25,135 p.]

Cet ouvrage et celui de Duchesne mentionné plus haut existent aussi en anglais.

1. Les normes de l'AF'NOR ayant toutes le format de $21 \times 27 \mathrm{~cm}$, nous ne l'indiquons qu'une fois. La Canadian Standards Association, 235, chemin de Montréal, Ottawa, est dépositaire des normes françaises et des recommandations internationales. 


\section{CORRESPONDANCE}

Association franghise de noRMalisation, Norme NF Z 11-001 - Documents administratifs - Tête de lettre - Grand format $(21 \times 27)$ [4 p.] et Annexe intitulée Instruction pour les dactylographes sur l'exécution de la correspondance normalisée. Paris, février 1941. [2 p.]

- Norme NF Z 11-002 - Documents administratifs - Tête de lettre Petits formats. Mai 1941. [4 p.]

Cette norme et la précédente renferment plusieurs éléments que le eorrespondant canadien aurait intérêt à adopter, tout en respectant les usages nord-américains essentiels de la correspondance.

Bernaténé, Henri, Le Secrétariat de direction. $2^{\mathrm{e}}$ éd., Louvain, Edditions d'organisation, 1963. [15.5 × 24, 148 p.]

Chaffurrin, Louis, Le Parfait Secrétaire. Nouv. éd., Paris, Larousse, 1954. $[11 \times 17.5,474$ p. $]$

Rideau, Marcel, Précis de correspondance commerciale - Courrier, Classement, Fiches. $4^{e}$ éd., Paris, Dunod, 1963. [20.5 × 27, 186 p.] Ouvrage inserit sur la liste des manuels adoptés par la Ville de Paris.

Verdeyen, Paula, Code de la secrétaire sténodactylographe et du correspondant. Tome I: Le Courrier de la parfaite sténodactylographe. Bruxelles, Baude, 1951. [13.5 x 20, 246 p.] - Tome II : Formation psychologique et professionnelle — Pratique du secrétariat. Bruxelles, Baude, et Paris, Editions scientifiques et littéraires, 1954. [317 p.]

\section{DICTIONNAIRES et Grammaires}

Benac, Henri, Dictionnaire des synonymes. Paris, Hachette, 1956. [12.5 × 20, 1026 p.]

Cassell's New French-English English-French Dictionary, Canadian Edition Incorporating Canadian Usage. Completely revised by Denis Girard with the Assistance of Gaston Dulong, Oliver Van Oss and Charles Guinness. Londres, Cassell \& Co. Ltd., 1962. [14.5 × 21.5, 1417 p.]

Dictionnaire qui renferme des termes exprimant des réalités eanadiennes.

Daviault, Pierre, Langage et Traduction. Ottawa, l'Imprimeur de la Reine, 1961. [16 × 24, 397 p.] Refonte et réunion des trois ouvrages $L ' E x p r e s-$ sion juste en traduction, Questions de langage et Traduction.

$L$ 'auteur y étudie méthodiquement une foule de termes anglais particulièrement difficiles à traduire et offre des équivalents français que l'on chercherait parfois en vain dans les dictionnaires de traduction ordinaires. Indispensable au rédacteur autant qu' 'au tradueteur.

Grevisse, MaUrice, Le Bon Usage - Grammaire française avec des remarques sur la langue française d'aujourd'hui. $8^{\mathrm{e}}$ éd., Gembloux, Duculot, 1964. [14 × 21, $1192 \mathrm{p}$.

HaNse, Joseph, Dictionnaire des difficultés grammaticales et lexicologiques. Bruxelles, Baude, et Amiens, Editions scientifiques et littéraires, 1949. $[14 \times 18.5,758 \mathrm{p}$.]

Thomas, Adolphe-V., Dictionnaire des difficultés de la langue française. Paris, Larousse, 1956. [13 x 19.5, XII-435 p.] 
Vinay, Jean-Paul; Daviault, Pierre; Alexander, Henry, Dictionnaire canadien - The Canadian Dictionary, français-anglais, anglais-français. Ed. abrégée, Toronto, MeClelland and Stewart Ltd., 1962. [13 × 19.5, 862 p.]

Premier dictionnaire bilingue conçu spécialement pour l'usager canadien.

\section{LINGUISTIQUE}

ACADÉmTe CANADIENNE-r'RANÇAISE, Bulletin de linguistique. Montréal, mensuel. $[21.5 \times 28]$

Commencée en janvier 1957, cette série a été remplacée à compter d'octobre 1962 par Mieux dire, publication présentée ci-après. L'Académie canadienne-franȩaise a recommencé à publier un bulletin mensuel de format réduit [14 x 21.5] en janvier 1965 .

Ministère des Affatres culturelles, OfFice de la langue frangaise, Mieux dire. Québec, mensuel. [21.5 × 28]

- , Norme du français écrit et parlé au Québec. Québec, 1965. [14.5 × 22, $12 \mathrm{p}$.

Le seul emploi du mot norme dans le titre révèle bien I'importance que ce document a pour le rédacteur canadien-français. Les dernières pages, qui sont consacrées aux anglicismes, nous semblent plus que toutes autres propres à attirer l'attention du grand publie sur la détérioration à laquelle le français est exposé au Canada.

Société Radio-Canada, Comité de linguistique, Fiches. Montréal, bimestrielles. $[7.5 \times 12.5]$

Ces fiches sont préparées à l'intention à la fois du personnel de Radio-Canada et du public. La première série date de novembre 1960. Le Comité publie en même temps un bulletin (bimestriel aussi) intitulé $C^{\prime}$ 'est-à-dire, qui complète avantageusement les fiches.

\section{Elaboration des PÉRIODIQUES et autres publications}

\section{Abréviation des titres}

american Chemical Society, Chemical Abstracts Service, List of Periodicals. Easton (Pa.), 1961. [20.5 x 26.5, 397 p.]

Les pages $12 \mathrm{~J}$ à $19 \mathrm{~J}$ renferment les abréviations des termes qui entrent dans les titres des périodiques. Un bref supplément à cette liste a'abréviations a été publié dans les Chemical Abstracts, 61 (juillet-décembre 1964): $4 \mathrm{~J}$.

Association françatse de normalisation, Norme NF Z 44-002 - Code d'abréviation des titres de périodiques en langue francaise. Paris, mai 1944. [16 p.]

CeNTre National DE LA RECHeRche screntifique, Liste des périodiques analysés par le Bulletin signalétique, $1^{\text {Te }}$ et $2^{e}$ parties et de leurs abréviations. Paris, 1959. [21 × 26.5, 54 p.]

Une nouvelle édition de cette liste doit paraître prochainement.

ORganisation INTERNationaLe DE NORMaLisation (ISO), Recommandation ISO R4 - Code international pour l'abréviation des titres de périodiques. $1^{\text {re }}$ éd., Genève, mars 1954. [21 x $29.5^{2}, 4$ p.]

2. Les recommandations de l'ISO ayant toutes le format de $21 \times 29.5 \mathrm{~cm}$, nous ne l'indiquons qu'une fois. Toutes les recommandations mentionnées dans la présente bibliographie existent aussi en anglais et en russe. 


\section{Manchette bibliographique}

Association Française de normalisation, Norme NF Z 44-003 - Manchette bibliographique de revue. Paris, avril 1954. [3 p.]

ORganisation internationale DE NORMaLisation, Recommandation ISO R30 Manchette bibliographique. $1^{\mathrm{re}}$ éd., Genève, décembre 1956. [4 p.]

\section{Ouvrages généraux}

Ministère des Finances et des Affatres économiques, Service centrad d'orgaNISATION ET MÉTHODES, L'Elaboration des imprimés administratifs. Paris, [1964 ?] [20.5 × 26, 118 p.] Comprend quatre parties: Comment concevoir un imprimé; Comment dessiner un imprimé; Connaissances techniques: papier, modes d'impression; Mise en cuvre.

Publication qui traite de l'aspect matériel seulement des imprimés administratifs et non de leur rédaction.

Organisation des Nations Unies pour l'Education, la science et la culture (UNESCO), Code du bon usage en matière de publications scientifiques. Rédigé par le Comité de liaison FID/ICSU/IFLA/ISO/UNESCO ${ }^{3}$, publié et diffusé par l'UNESCO. Applicable «aux sciences exactes et naturelles et à la technologie ».

PoIRIER, LÉANDRE, O.F.M., Au Service de nos écrivains - Directives pratiques pour publications. Nouv. éd., Montréal, Fides, 1964. [14 × 20.5, IX193 p.]

Le texte intégral du Code a été reproduit dans le Bulletin do la Société do chimie biologique, 45.2-3 (1963): 181-184.

\section{Présentation des articles}

ORganisation INTERNATIONALE DE NORMALISATION, Recommandation ISO R215 Présentation des articles de périodiques. $1^{\text {re }}$ éd., Genève, novembre 1961. [4 p.]

\section{Présentation des périodiques}

Association franghaise de normalisation, Norme NF Z 41-001 - Présentation des revues. Paris, septembre 1942 (en revision). [8 p.]

Organisamion INTERNationale DE NORMaLISATION, Recommandation ISO R8 Présentation des périodiques. $1^{\text {re }}$ éd., Genève, octobre 1955. [8 p.]

\section{Références bibliographiques}

Organisation INTERNationale DE NORMALISATION, Recommandation ISO R77 Références bibliographiques - Eléments essentiels. $1^{\mathrm{re}}$ éd., Genève, décembre 1958. [7 p.] Renferme de nombreux exemples.

\section{Sommaires, Analyses et Résumés}

Logie, Paul \& Mouchez, Phmippe, La Technique du résumé. Paris, Cujas, 1962. $[14 \times 19,102 \mathrm{p}$.

3. $F I D=$ F'édération internationale de documentation; ICSU $=$ International Council of Seientific Unions (Conseil international des unions scientifiques); IFLA = International Federation of Library Associations; ISO = International Organization for Standaräization; UNESCO = United Nations Edueational, Scientific and Cultural Organization. 
Organisation des Nations Unies pour l'Éducation, la science et la culture (UNESCO), Guide pour la rédaction des résumés d'auteurs ${ }^{4}$ — Document NS/37.D 10a, préparé et revisé périodiquement par l'UNESCO. Ce texte a été reproduit dans le Bulletin de l'Institut international du froid, 43.2 (1963) : 609-613.

Organisation Internationale de noRmalisation, Recommandation ISO R.18 -

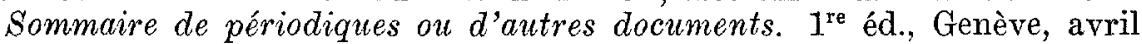
1956. [3 p.]

- , Recommandation ISO R214 - Analyses et résumés d'auteurs. $1^{\text {re }}$ éd., Genève, novembre 1961. [4 p.]

\section{PROTOCOLE}

SERREs, JEAN, Manuel pratique de protocole. Nouv. éd. refondue, augmentée et mise à jour, Vitry-le-François (Marne), Editions de l'Arquebuse, 1965. [11.5 x 18,543 p.]

\section{Rédaction des RAPPORTS}

Blanchard, $\mathrm{C}^{1}$ Justin, Les Modèles du gendarme - Recueil de modèles types de procès-verbaux, de rapports et de dossiers entièrement traités, suivis de conseils pratiques. Clamart (Seine), Degrives, 1948. [18 x 27, 295 p.]

- Le Deuxième Livre de modèles - Recueil de modèles types de procèsverbaux, de rapports et de dossiers entièrement traités. Clamart (Seine), Degrives, 1962 . [21 x 27, 303 p.]

Bousquié, Georges, Comment rédiger vos rapports. Paris, Editions de l'entreprise moderne, 1957. [13.5 x 21, $120 \mathrm{p}$.]

Desonay, Fernand, Le Rapport - Comment l'élaborer, comment le rédiger. «Bien écrire et bien parler», $3^{\mathbf{e}}$ éd., Bruxelles, Baude, et Amiens, Editions seientifiques et littéraires, 1949. [14 x 18.5, 294 p.]

Desseaux, Pierre, Rapports et Comptes rendus. Paris, Editions Hommes et Techniques, 1959. [13.5 x 21, 117 p.]

Georgrn, Charles, Cours de rédaction des rapports. $27^{\mathrm{e}}$ éd., Paris, Eyrolles, 1962. [16 $25, \mathrm{X}-287 \mathrm{p}$. $]$

Roumagnac, J., Rédaction de rapports, comptes rendus, procès-verbaux. Paris, Foucher, 1956. [21 x 27, 150 p.]

* Soctété nationale des chemins de fer fRançass, Cours pratique de composition française et de rédaction administrative. $4^{\mathrm{e}}$ éd., Paris, 1959. [18 $\mathrm{x} 23,186 \mathrm{p}$.

WaCKermann, Gabriel \& Wirhelm, A., Initiation à la technique du rapport. 《Bibliothèque de l'enseignement technique », $3^{\mathrm{e}}$ éd. revue et augmentée, Paris, Dunod, 1965. [16 x 25, 332 p.]

\section{STYLE ADMINISTRATIF}

* Catherine, RoBert, Le style administratif. Nouv. éd., Paris, Albin Michel, 1961. [12 $18.5,170$ p. $]$

4. Voir au sujet de ces publications le Courrier de la normalisation, 169 (janvier-février 1963) : 44-45 et surtout 181 (janvier-février 1965) : 99-105.

* Les ouvrages marqués d'un astérisque traitent aussi de la correspondance. 
* Datain, Jean, L'Art d'écrire et le Style des administrations. Ed. complétée, Paris, Charles-Lavauzelle \& $\mathrm{C}^{\mathrm{ie}}, 1960$. [14 $\mathrm{x} 21$, XII-162 p.]

Georarn, René, Le Code du bon langage. Paris, Editions sociales françaises, 1960. [13 $122,231 \mathrm{p}$. $]$

- ,Consultations de grammaire, de vocabulaire et de style. Paris, Editions sociales françaises, 1964 . [13 $\times 22,227$ p.]

- L'Inflation du style. Paris, Editions sociales françaises, 1963. [13 x 22, 199 p.]

— , Le Langage de l'administration et des affaires. $7^{\mathrm{e}}$ éd., Paris, Editions sociales françaises, 1954. [13 x 22, 208 p.]

Hot, AlFred, Traité de style administratif. Montpellier, Causse-Graille et Castelnau, 1949. [15.5 x 24.5, 171 p.]

\section{STYLE JUDICIAIRE}

Francoz, P., La Rédaction des actes et des contrats. «Manuels de législation et de pratique sociale », Paris, Editions sociales françaises, 1955. [11.5 x 18, 200 p.]

Lambert, Lours, Formulaire expliqué de l'enquête pénale. Publié par PoliceRevue, 55, rue de Rivoli, Paris, 1965 [250 p.]. Cet ouvrage s'adresse aux «candidats aux fonetions d'officier de police judiciaire ». Signalé par M. Pierre Mimin, dans Défense de la langue française, $\mathrm{n}^{\circ} 29$ (septembre 1965) : 31-32.

— , Le Style de l'information pénale - Manuel de procédure écrite à l'usage des commissaires de police et des autres magistrats. $3^{\mathrm{e}}$ éd. complétée et mise à jour, Lyon, Desvigne, 1953.

Mimiv, Pierre, Le Style des jugements - Vocabulaire, Construction, Dialectique, Forme juridique. $4^{\mathrm{e}}$ éd., Paris, Librairies techniques, 1962. $[14 \times 22.5,422 \mathrm{p}$.

\section{TRANSLITTERATION}

Association Frangaise DE norMalisation, Fascicule de documentation FD Z $\mathbf{n}^{\circ}$ 46-001 - Translittération des caractères cyrilliques. Paris, janvier 1956.

Organisation internationale DE NORMalisation, Recommandation ISO R233Système international pour la translittération des caractères arabes. Genève, 1961. [8 p.]

— , Recommandation ISO R9 - Système international pour la translittération des caractères cyrilliques. Genève, 1954. [ 7 p. $]$

L'ISO a aussi annoncé des projets pour la translittération d'au moins deux autres types de earactères, soit les:

Projet ISO no 315 - Transitttération des caractères grecs en caractères latins et

Projet ISO no $379-$ Transititération de l'hébreus.

\section{TYPOGRAPHIE - Présentation des manuscrits}

Assoctation française de norufalisation, Norme NF Z 09-002 - Signes de corrections dactylographiques. Paris, mai 1945. [1 p.] 
Gouriou, Chardes, Mémento typographique. Paris, Hachette, 1961. [21 × 27, XI-131 p.]

Codification des usages typographiques de la maison Hachette. S'adresse à la fois à. l'auteur, à l'éditenr et à l'imprimeur.

Laloue, Albert, Règles typographiques (A l'usage des opérateurs). Paris, Edition de l'Ecole d'opérateurs, 1953 . [13.5 x 21, 192 p.]

Transposition des règles du Code typographique à l'intention des opérateurs, dit en substance l'auteur dans la préface. L'ouvrage renferme un index alphabétique des matières.

Lecerf, PIerre, Manuel pratique du typographe. $2^{\mathrm{e}}$ éd., Paris, Dunod, 1963. $[12 \times 18,495 \mathrm{p}$.

Organisation des Nations Unies pour l'Éducation, la science te la culture (UNESCO), Guide de présentation des manuscrits - Projet de guide bibliographique. Paris, 1956. [21 x 27, 113 p., multicopié.]

Texte provisoire préparé par l'UNESCO pour ses propres services.

Pernin, André, Composition typographique. $1^{\text {re }}$ éd., Paris, Eyrolles, 1957. [16 × 25,484 p.]

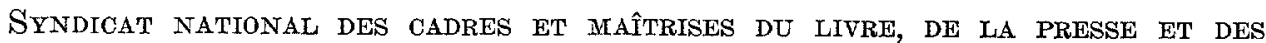
INDUSTRIES GRAPHIQUES (SNCML), Code typographique - Choix de règles à l'usage des auteurs et des professionnels du livre. $8^{\mathrm{e}}$ éd., Paris, 1965. $[13.5 \times 21.5,124$ p. $]$

Codification des règles typographiques françaises établie en 1926 par une commission spéeiale de l'Amicale des directeurs, protes et correeteurs d'imprimerie de France. Les ehapitres s'intitulent Abréviations; Grandes capitales, bas de casse, petites eapitales; Italiques; Division des mots et coupures entre les mots; Nombres; Notes et références bibliographiques; Ponotuation et signes.

Publieation essentielle au Canada français où l'on est si exposé à confondre les usages français avec les usages anglais. Il suffit en effet d'observer les multiples façons dont certains abrègent l'adjectif numéral ordinal deuxième pour conclure à l'utilité que peut avoir une règle en ce domaine. Nous avons relevé les abréviations Zième, Zème, Zme, Ze., au lieu de $2 e$, abréviation recommandée par le Code (p. 14, art. 21).

«S'il nous était permis d'émettre un vœu» éerivait M. J. Laudats dans l'Avantpropos de la sixième édition (1957) du Code, «nous souhaiterions le voir recommander par l'Enseignement technique dans toutes ses écoles, pour que, de la dactylographe jusqu'au linotypiste, tous parlent le même langage. » La réalisation de ce vœu ne serait-elle pas avantageuse à plus forte raison au Canada ? On pourrait rendre le Codo plus facilement utilisable en $y$ ajoutant en annexe une liste d 'exemples canadiens touehant l'emploi de la majuscule ainsi que les abréviations françaises de nos principales unités de mesure anglo-saxonnes 6.

Il $n$ 'existe pas en français, autant que nous sachions, d'ouvrages aussi complets que les guides anglais que publient les grands éditeurs d'ouvrages scientifiques, MeGrawHill, par exemple, et même des associations telles que l'American Institute of Biological Sciences 7. Le rédacteur scientifique canadien-français trouvera les mêmes éléments, une partie au moins, dans divers documents du genre de ceux que nous avons énumêrés mais également dans les conseils ou avis présentés au début de divers

5. Alors président du SNCML.

6. Voir ci-dessous, p. 40, ce que nous disons au sujet de la norme canadienne Z85-1963.

7. Voir The McGraw-Hill Author's Book. Toronto, McGraw-Hill, 1955 [22.5 x 15, 88 p.] et le Style Manual for Biological Journals, 2nd ed., Washington, American Institute of Biological Sciences, 1964. [21 x 18, $117 \mathrm{p}$. 
périodiques seientifiques français. Les éditeurs français aussi offrent aux auteurs des instructions concernant l'établissement des manuscrits. Les Annales de l'Institut national de la recherche agronomique, par exemple, fournissent en tiré à part aux auteurs d'articles des Recommandations très détaillées et très instructives. [17 x 24, 7 p.]

\section{UNITES et Symboles}

Association FRançaise de nORMalisation, Norme NF K 10-02 - Symboles d'unités monétaires. Paris, novembre 1956. [4 p.]

Norme qui a pour objet de « fixer les symboles graphiques des monnaies des principaux pays du monde et leurs règles d'écriture» (p. 1 de la Norme). Elle est utilisable sans modification au Canada. Le symbole international du dollar canadien est \$CAN. Cependant, lorsqu'on utilise une machine à écrire ne portant pas le signe du dollar, ou une machine mécanographique ou un téléseripteur, les symboles CAN et SCAN sont aussi admis.

- Fascicule de documentation FD X $\mathrm{n}^{\circ}$ 02-050 - Principales unités de mesure américaines et britanniques. Paris, mai 1959. [14 p.]

Même si elles ont été préparées pour la France, les normes françaises NF X 02.003 à 02-105 et les fascicules de documentation $\mathrm{FD} X \mathrm{n}^{\circ}$ 02-106 et no 02-107, qui traitent des unités et des symboles, et la norme NF X 05-001, intitulée Vocabulaire de la statistique, ont cependant. leur utilité au Canada.

Canadian Standards Association, CSA Standard Z85-1963 - Abbreviations for Scientific and Engineering Terms (For use in text). Ottawa, décembre 1963. [15 $\times 22.5,15 \mathrm{p}$.]

Il est à souhaiter qu'une commission formée d'ingénieurs et de linguistes prépare un jour l'homologue français de cette norme, e'est-à-dire un texte qui établirait des abréviations françaises pour nos unités anglo-saxonnes. Ce texte pourrait ensuite être soumis à l'homologation de l'Office de la langue française du Québec et être diffusé dans les institutions d'enseignement.

Dayre, Jean \& Cassan, Michel, "Transunit»-Guide graphique pour la conversion des unités anglo-saxonnes et métriques. Paris, Eyrolles, 1954. [13.5 $\times 21,48$ p.]

Rennie, Peter John, Measure for Measure. $3^{\text {e }}$ éd., publication multicopiée $n^{\circ} 62-9$, Ottawa, Ministère des Forêts, 1963. [21.5 x 28, 43 p.]

Publication préparée pour servir avant tout dans la recherche forestière et le commeree des produits da bois. Renferme la conversion d'unités britanniques en unités américaines et métriques.

\section{VULGARISATION AGRICOLE}

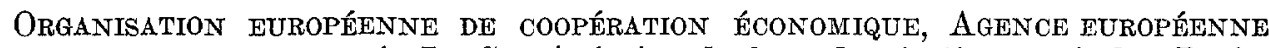
DE PRODUCTIVITÉ, Le Savoir-écrire de la vulgarisation agricole. Paris, 1956. $[16 \times 26,79 \mathrm{p}$.

Le lecteur aura sans doute remarqué que les normes et les recommandations internationales sont ici présentées sur le même pied. L'Organisation internationale de normalisation fait cependant toujours remarquer à la première page de couverture de ses recommandations que «Seules les normes nationales sont valables dans leurs pays respectifs ». Il ne se pose pas de problème à ce sujet au Canada français puisque les normes de la Canadian Standards Association sont publiées en anglais seulement.

David Fortin

Ottawa 